\title{
COMMUNICATION A LA TROISIEME CONFERENCE REGIONALE POUR JEUNES CHERCHEURS ${ }^{1}$
}

\author{
De l'absence de la «culture» du respect des textes juridiques en \\ matière d'exploitation et d'utilisation des ressources naturelles en \\ faveur de la population congolaise
}

\author{
Par le Professeur SANGO MUKALAY Adalbert ${ }^{2}$
}

\section{Introduction}

Beaucoup a été écrit sur la thématique de ma communication. Donc je ne reviens pas, ici, pour parler de l'exploitation et de l'utilisation des ressources naturelles, mais plutôt du pillage systématique de ressources naturelles comme moyens d'enrichissement illégal et sans cause en République Démocratique du Congo. Je me propose donc d'axer mon propos sur un principe subjectif, qui par la suite devient légal, et pouvant faire profiter à la population congolaise de ses richesses. Il s'agit de reconnaitre non seulement l'existence des textes légaux, en la matière et qui créent par ricochet un droit : le droit à l'information juridique, mais aussi d'y recourir pour sanctionner toute violation éventuelle.

La République Démocratique du Congo, comme tous les autres pays du monde, a ratifiédes textes déclaratifs ou légaux internationaux. Elle a su, aussi, intégrer certains d'entre eux dans son arsenal juridique pour la protection de ses citoyens. Force est de constater, hélas, que l'impact de ces textes, sur la vie de la population est presqu'inexistant. Il en va, ainsi, de ceux relatifs à l'exploitation et l'utilisation des ressources naturelles. La RDC possède un code minier, un code forestier, des textes en matière hydrique et j'en passe.

La question qui se pose, en effet, est celle de savoir « pourquoi les prescrits de tous ces textes ne sont respectés ou ne le sont qu'en partie? »Quelles sont les stratégies, prévues par ces mêmes textes, devraient être adoptées pour leur mise en pratique.

Il ressort de l'analyse générale de la situation, en la matière, qu'il existe un paradoxe saisissant entre la garantie juridique et les attentes de la population; et surtout entre l'exploitation irrationnelle des matières naturelles du pays et le souci de leur protection pour les générations futures. Quelle est la cause de ce comportement et que faut-il faire? Cette question nous fait entrer dans le vif du sujet sous examen.

1 Dar es Salaam, du 27 au 28 octobre 2014.

2 Professeur à la faculté de Droit de l'Universitéde Lubumbashi (RDC). 
1. La garantie juridique en matière d'exploitation et d'utilisation des ressources naturelles en RDC : nécessité d'une information juridique

Il sera question dans ce point de réaffirmer l'existence des textes légaux et la responsabilité de l'élite, qu'elle soit politique ou culturelle, dans leur non application.

A cet effet, il sied de recourir à une question, pour comprendre encore mieux ce paradoxe : "Pourquoi y-a-t-il encore tant de pauvreté dans le monde alors que nous avons aussi bien les connaissances que les moyens de l'éradiquer de la surface de la terre?» ${ }^{3}$ Plusieurs paramètres sont à la base de cette situation. Il existe, dans le chef de l'élite de différents Etats africains, une culture savamment entretenue qui consiste à promouvoir des «fausses priorités» ${ }^{4}$ qui ne profitent qu'à elle. Ces «(...) fausses priorités s'enracinent souvent dans des avantages acquis qui empêchent l'éradication de la pauvreté. C'est qu'il y a des élites qui bénéficient, directement ou indirectement, des dépenses militaires, de projets somptuaires ou encore du grand business, que les pauvres sont marginalisés $\rangle^{5}$, au risque de ne pas jouir des richesses de leur sol et de leur sous-sol.

Ce type d'attitude fait naître une idéologie du gain à tout prix, par la minorité régnante au détriment de la majorité, consciemment maintenue dans une forme d'analphabétisme qui ne dit pas son nom. Cela, surtout «lorsque les moyens d'exprimer publiquement leurs doléances se trouvent contrecarrés par des politiques autoritaires» ${ }^{6}$ et mêmes démagogiques.

Pour transcender ce paradoxe, il est du devoir des gouvernants de promouvoir le droit à l'information. Pas n'importe quelle information, mais une information juridique, qui constitue la règle et non l'exception.

Le droit à l'information représente donc, sous cet aspect, un droit plus général du citoyen à participer à la vie politique et à entretenir des rapports avec une administration publique impartiale, qui agit sur la base du principe de transparence. Cependant ce principe n'a pas encore atteint son actualisation en RDC. De ce point de vue, la démocratie, dans un pays, se traduit par la possibilité de participation de ses citoyens à la vie culturelle, sociale et politique, à travers la connaissance et la compréhension des contenus de l'action de l'État, des raisons qui sous-tendent cette action et des modalités avec lesquelles elle est réalisée.

L'information juridique comme processus de communication entre les institutions et les citoyens se concrétisent par le respect des règles et des structures del'Etat. L'information juridique se situe dans le processus d'un ample développement social, qui implique différents domaines. Comme droit subjectif, elle a à coeur le rapport entre l'État et les citoyens. L'État, en tant qu'expression et protecteur de l'intérêt de la collectivité, doit se renouveler, institutionnellement, pour être fiable et crédible, et les citoyens devenant ainsi acteurs principaux de ce changement social.

3 Albert LONGCHAMP, Face au fossé grandissant entre riches et pauvres. Toutes les religions en appellent à la justice, Congo Afrique, septembre 2003, n³77, p. 413.

4 Ibid.

5 Ibid., p.414.

6 Ibid. 
L'action des pouvoirs publics doit tendre vers la transparence et l'écoute, l'efficacité et l'efficience. Mais la réalisation de ce processus doit nécessairement être accompagnée par un changement culturel, à travers soit les méthodes de travail à l'intérieur des institutions et par leur attitude vis-à-vis decette partie d'utilisateurs conscients de leurs propres droits et devoirs en tant que citoyens.

La démocratie est le gouvernement du pouvoir visible et non opaque. La publicité est un principe fondamental de l'État constitutionnel, alors que le secret est une exception qui doit être justifiée et limitée dans le temps. Un gouvernement démocratique est un gouvernement d'un pouvoir qui rend publiques ses actions. Il est un pouvoir qui informe ses citoyens.

Pour la RDC, la garantie de la jouissance, par la population, de ses richesses naturelles et de leur protection contre tout vandalisme dans leur exploitation se trouve dans la constitution. Ce sont les articles 54, 55, 56 et 58 de ladite Constitution. Passons en revue et très rapidement ces dispositions constitutionnelles :

Article 54 :

«Les conditions de construction d'usines, de stockage, de manipulation, d'incinération et d'évacuation des déchets toxiques, polluants ou radioactifs provenant des unités industrielles ou artisanales installées sur le territoire national sont fixées par la loi.

Toute pollution ou destruction résultant d'une activité économique donne lieu à compensation et/ou à réparation.

La loi détermine la nature des mesures compensatoires, réparatoires ainsi que les modalités de leur exécution.»

Sans vouloir forcer le texte de cet article, l'alinéa 2 garantit, à mon avis, une certaine utilisation adéquate des milieux d'exploitation des ressources naturelles.

Article 55 :

«Le transit, l'importation, le stockage, l'enfouissement, le déversement dans les eaux continentales et les espaces maritimes sous juridiction nationale, l'épandage dans l'espace aérien des déchets toxiques, polluants, radioactifs ou de tout autre produit dangereux, en provenance ou non de l'étranger, constitue un crime puni par la loi.»

Article 56 :

«Tout acte, tout accord, toute convention, tout arrangement ou tout autre fait, qui a pour conséquence de priver la nation, les personnes physiques ou morales de tout ou partie de leurs propres moyens d'existence tirés de leurs ressources ou de leurs richesses naturelles, sans préjudice des dispositions internationales sur les crimes économiques, est érigé en infraction de pillage punie par la loi.» 
La conséquence de la violation de ces dispositions est sanctionnée par l'article 57 de la même Constitution, qui dispose : «Les actes visés à l'article précédent ainsi que leur tentative, quelles qu'en soient les modalités, s'ils sont le fait d'une personne investie d'autorité publique, sont punis comme infraction de haute trahison.»

La Constitution de la RDC, tout en reconnaissant le droit de ses citoyens congolais de jouir de leurs richesses naturelles, érige en infraction tout autre comportement contraire à la loi, c'est-à-dire toute violation des dispositions constitutionnelles établissant ce droit. Mais hélas! La vérité étant têtue, il existe des comportements déviationnistes dans le chef de l'élite qui prend des lois sans pour autant les respecter: l'inadéquation entre l'exploitation et utilisation des matières premières, le problème de pillage, l'existence et la facilitation de l'exploitation illégale, l'impunité à l'égard des violeurs des textes légaux et réglementaires existants.

Tout cela est possible, parce que la grande majorité de la population reste sous-informée non seulement de leurs droits, mais aussi de la possibilité que le droit objetif laisse au citoyen de se plaindre de la mauvaise gestion de son patrimoine national. D'où le citoyen reste maintenu, sciemment, dans une forme d'analphabétisme.

L'information juridique comprend un vaste champ d'informations qui proviennent soit de l'État soit de différentes administrations. Le premier domaine est celui des pouvoirs publics, plus complexe, parce qu'il implique inévitablement la sphère politique: d'où la communication publique devient l'instrument d'expression de la démocratie. En ce qui concerne les autres administrations, l'activité d'informer assume les caractéristiques de « communication de service »: on parle, alors, d'un devoir des administrations vis-à-vis des citoyens.

\section{Le manque d'information et le déficit de communication, comme facteurs déterminants du détournement des moyens mobilisés pour le développement}

La situation de mise à l'écart des populations de la gestion des fruits de l'exploitation de leurs richesses naturelles constitue un chemin qui ne mène nulle part ${ }^{7}$. Le Droit à l'information est garanti par la constitution du 18 février 2006 telle que modifiée par la loi n 11/002 du 20 janvier 2011 portant révision de certains articles:

- à l'article 23: elle accorde à toute personne la liberté d'expression;

- à l'article 24: elle reconnaît à toute personne le droit à l'information et lui garantit la liberté de la presse;

- à l'article 23: elle consacre, pour toute personne, le droit à la liberté d'expression et stipule que « ce droit implique la liberté d'exprimer ses opinions ou ses convictions notamment par la parole, l'écrit et l'image, sous réserve de la loi, de l'ordre public, de bonnes mœurs ». Cette disposition n'explique pas, néanmoins, les modalités d'exercice de ce droit.

7 Jean-Marc ELA, L'Afrique des villages, Karthala, Paris 1982, p.82. 
- à l'article 24, tout en garantissant le droit à l'information, elle explicite, de manière implicite, les modalités d'exercice de ce droit quand il disposeque «toute personne a droit à l'information ». Il s'agit ici de l'information en général. Néanmoins l'information qui m'intéresse et que j'aimerais développer, dans cette communication, et l'information juridique.

Dans la relation entre l'information juridique et la citoyenneté, les activités de l'information des institutions ont comme but la réalisation d'un rapport d'échanges, de participation et de confiance entre les citoyens et l'apparat institutionnel. Le rôle du citoyen est ainsi reconsidéré: il devient sujet actif et participant des choix et de la définition de projets et initiatives qui répandent le sens civique et renforcent le sens d'appartenance à la cité.

Il faut déterminer les rôles et les responsabilités de différents sujets qui collaborent et qui partagent la gestion des situations déterminées et leur connaissance par les citoyens.

L'information juridique revêt donc une fonction: celle de contribuer à la réalisation de l'intérêt commun, à travers l'ensemble des connaissances et d'expériences, fruit du concours de plusieurs voix, d'une interaction de rôles, de compétences et de responsabilités différentes mais toujours aussi rapprochéespar le même objectif: le bien public. Il peut, en outre, développer la valeur de l'appartenance à la citoyenneté et favoriser l'éducation à la démocratie: le bien commun, compris non pas comme une déduction éthique, théorique ettranscendantale, mais comme une construction logique résultant d'un système partagé et garanti de droits et devoirs civiques des citoyens.

Dans le rapport entre l'information juridique et la démocratie, le droit à l'information juridique représente un fondement indispensable de la démocratie, antécédent logique de tout autre droit.Quand bien même les textes constitutionnels omettent de l'énoncer explicitement, la législation et la jurisprudence des Cours et Tribunaux y ont toujours pourvu dans le monde entier.

Dans la société actuelle, dans laquelle il y n'a pas de rapport ou de relation qui ne soit conditionné par les décisions des pouvoirs publics, il est juste de fournir au citoyen la possibilité de « connaître » pour récupérer une position d'égalité avec les organisations et organismes qui l'administrent et qui le dominent.

La science constitutionnaliste a procédé par étapes à l'élaboration du droit à l'information. Elle s'est tout d'abord imposé la liberté d'acquisition de l'information non plus seulement comme un profil passif de la manifestation de la pensée d'autrui, mais comme une situation subjective protégée. Ensuite a émergé la tentative d'en faire une illustration autonome, non pas fondée sur la règle relative à la liberté de manifestation de la pensée, mais sur le principe de démocratie.

Dans cette perspective la liberté d'information est construite comme l'instrument juridique de garantie et de discipline de la connaissance des citoyens en vue de leur participation effective à la gestion de la chose publique et de leur contrôle des activités des pouvoirs publics.

Le célèbre adage, selon lequel l'ignorance de la loi ne serait ni pardonnable ni considéré comme une circonstance atténuante éventuelle, bien qu'étant un des principes cardinaux du 
droit, il est peut être un des « axiomes » dont transparaît le fait que l'on n'ait pas mis en évidence l'obligation civile à l'information et à la vulgarisation législative dans son acception la plus générale en République Démocratique du Congo.

L'exercice de la démocratie par l'information, ou mieux l'information pour la démocratie doit avoir comme objectif la diffusion de toutes ces nouvelles et informations pouvant permettre au citoyen, quelque soit son statut ou son niveau d'instruction, de connaître ses droits et ses devoirs d'une manière adéquate, mais aussi de connaitre quels sont les services et les obligations relevant des relations quotidiennes avec l'Administration publique.

A l'affirmation du droit à l'information, l'on ne peut pas ne pas faire correspondre, à charge de l'État, un devoir d'information, une politique active de la part des pouvoirs publics pour favoriser la connaissance des actes et des documents, et d'une façon générale, des données en leur possession, par les citoyens; en d'autres mots le devoir de diffuser toutes les mesures nécessaires pour que les citoyens et organisations sociales puissent être informées en vue d'exercer leur droit-devoir de participation à la vie politique et sociale.

Il existe un devoir public de consentir à tous les citoyens, et ce gratuitement, la possibilité de connaître les lois, les sentences et les actes réglementaires à travers les nouveaux instruments informatiques et télématiques aussi, et de les rendre aisément connaissables et trouvables par toutes les composantes de la collectivité étatique.

Un citoyen informé est essentiel pour la création d'un processus démocratique et décisionnel sain. Et plus il sait des autorités qui le gouvernent et mieux il sera gouverné. Le devoir indéfectible du législateur est celui de faire en sorte que le commandement impératif contenu dans la loi ait le maximum de vulgarisation entre les citoyens afin d'amplifier la possibilité d'une effective et consciente réalisation.

\section{Conclusion}

La connaissance des lois et des actes parlementaires est une ressource à promouvoir à tout prix, parce qu'elle représente une base essentielle pour la construction d'une conscience démocratique, civile, sérieuse et consciente. Il faut toutefois souligner que le droit à l'information, avant d'être un principe constitutionnel, est d'abord une situation subjective, c'està-dire qu'il est lié à un droit originaire de l'homme : le droit à la vie.

Il est clair que la juridicisation de ce droit crée un problème, celui de sa politisation. Car le reconnaitre et ne pas le faire exercer rend plus commode la situation des décideurs.Car quelle est la proportion des Congolais qui sait que le code minier renferme des clauses sociales en leur faveur? Combien sont conscients de l'irrémédiabilité de la destruction des forêts par l'abattage illégal des arbres? Combien se rendent compte que l'eau de certains ruisseaux qu'ils boivent et dont ils se lavent est polluée et a des conséquences insoupçonnées et insoupçonnables sur leur état de santé?

Des réponses à toutes ces questions, non exhaustives par ailleurs, dépend le degré de connaissance de la majorité de la population de leurs droits sur l'utilisation, par l'élite 
culturelle et politique régnante, des ressources naturelles que leur sol et leur sous-sol regorgent. 